\title{
Factors affecting the development of night time temperature rhythms
}

\author{
M R Lodemore, S A Petersen, M P Wailoo
}

\begin{abstract}
The rectal temperature of 26 infants between 6 and 16 weeks old was monitored continuously for one night each week. Rectal temperature always decreased with sleep but the minimum temperature attained changed with age. Some time between 8 and 16 weeks old the minimum sleeping rectal temperature decreased abruptly from around $36.8^{\circ} \mathrm{C}$ to around $36.4^{\circ} \mathrm{C}$. This change was complete within one week and did not normally revert unless the infant became ill.

Some infants changed as early as 8 weeks old, others not until 16 weeks. Breast fed infants changed significantly earlier than bottle fed infants. Girls changed significantly earlier than boys. First born infants changed significantly earlier than second or subsequent infants. Early changers were significantly more likely to be sleeping lateral or supine, and to have older mothers. They tended to come from more affluent families. There was no association between the time of change and the thermal environment in which the infant slept or the number of episodes of minor illness in the early weeks of life.
\end{abstract}

(Arch Dis Child 1992;67:1259-61)

When infants are first brought home their body temperature changes little over the course of the light-dark cycle and for the first weeks of life their rectal temperature decreases to about $36.8^{\circ} \mathrm{C}$ with sleep, whether this occurs by day or by night. In a previous cross sectional study ${ }^{1}$ we showed that at around 10-12 weeks of age rectal temperature begins to decrease further with sleep at night than during the day, a night time pattern which persists into adulthood. Within two hours of bedtime rectal temperature decreases to less than $36.5^{\circ} \mathrm{C}$ and then remains low for a few hours before a gradual warming to around $37^{\circ} \mathrm{C}$ before waking. ${ }^{2}$

There appeared, however, to be considerable individual differences in the age at which this pattern appeared. This paper reports longitudinal studies of individual infants which confirm these differences, and shows some factors affecting the timing of development of night time temperature rhythms.

\section{Subjects and methods}

Thirty normal healthy full term infants were recruited at or just after birth by liaison with general practitioners and health visitors in Leicestershire. Parents gave informed consent to weekly overnight recordings of body temperature from 6 to 16 weeks of age. Basic perinatal data were collected at the time of recruitment and parents were provided with a diary to record prospectively any episodes of illness in the infant.

Once each week from 6 weeks' old the infant was visited at home in the evening, weighed naked, and data collected on the current feeding regimen, other aspects of parental care, and the health of the infant and family. Probes were then attached to record body temperature. A soft probe, inserted $5 \mathrm{~cm}$ from the anal margin, recorded rectal temperature and a skin thermistor measured surface temperature on the skin. Only rectal temperature data are described here. A third probe measured ambient temperature near the infant. All three probes were connected to a Grant Squirrell data logger, set to sample at one minute intervals throughout the night. These techniques are safe, ${ }^{1}$ and full ethical approval was obtained for the study.

Parents were asked to keep a detailed diary of all events throughout each night of recording, including feeds, nappy changes, periods of waking, and administration of any drugs. Note was also made of infant's clothing and bedding so that a thermal insulation or 'tog' value could be calculated according to tables provided by the Shirley Institute (Manchester).

The next day the data loggers were collected and the data downloaded to a computer. They were analysed for technical problems such as loss of probes and only unblemished data were analysed further. For each night of recording rectal temperature was abstracted at 30 minute intervals from one hour before bedtime until nine hours later.

\section{Results}

Twenty six of the 30 subjects yielded sufficient data to be certain of the individual pattern of development, a total of 246 nights of recording. For the remaining four infants data were not available for one or more weeks around the critical age for a variety of reasons. The mean (SD) gestational age of the 26 infants was 39.9 $(1.5)$ weeks, with all but one born between 38 and 42 weeks and none before 36 weeks. The birth weight averaged $3471(485) \mathrm{g}$ and 13 were boys. The average maternal age was $27 \cdot 3(4 \cdot 6)$ years. Six of the infants had parents in occupational groups 1 or 2 , and seven in groups 4 or 5 , a social class distribution similar to that of the Leicestershire population as a whole. Nine were first children; three had two or more older siblings. 
CHANGES IN SLEEPING RECTAL TEMPERATURE WITH AGE

At all ages studied rectal temperature decreased with sleep at night reaching a minimum between one and four hours after bedtime, as reported previously. $^{12}$. The minimum temperature attained by any infant, however, changed with age. Figure 1 shows a typical example of individual development. The minimum rectal temperature decreased suddenly by $0.3-0.5^{\circ} \mathrm{C}$ between one week and the next, though this occurred at different ages in different subjects. Figure 2 shows how the minimum rectal temperature changed with age in five further infants. All 26 infants studied showed similar abrupt changes in minimum rectal temperature. As in our previous studies there was no significant change in the thermal environment after 6 weeks of age.

Once this abrupt transition to a lower minimum night time rectal temperature had occurred, the minimum remained low unless the infant was incubating an illness. Figure 3 shows data from all subjects normalised to the first week in which rectal temperature decreased below $36 \cdot 5^{\circ} \mathrm{C}$, which we have called the 'adult' pattern.

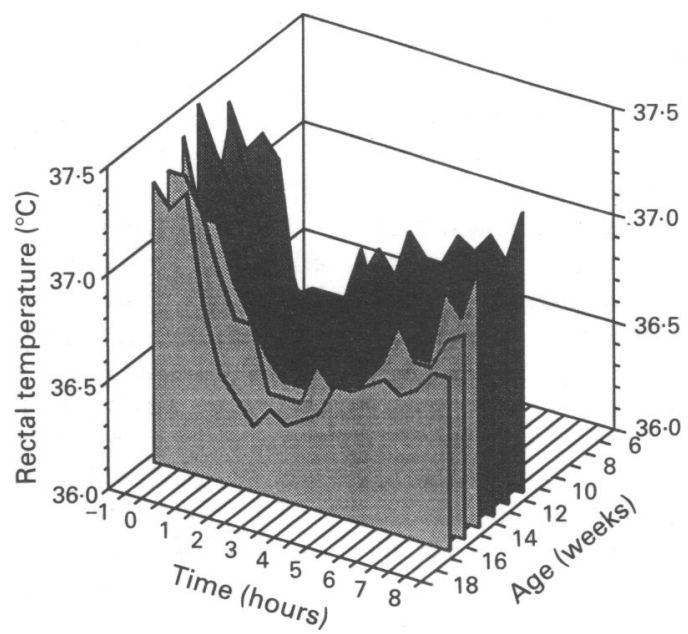

Figure 1 Pattern of rectal temperature change overnight at different ages in a typical infant.

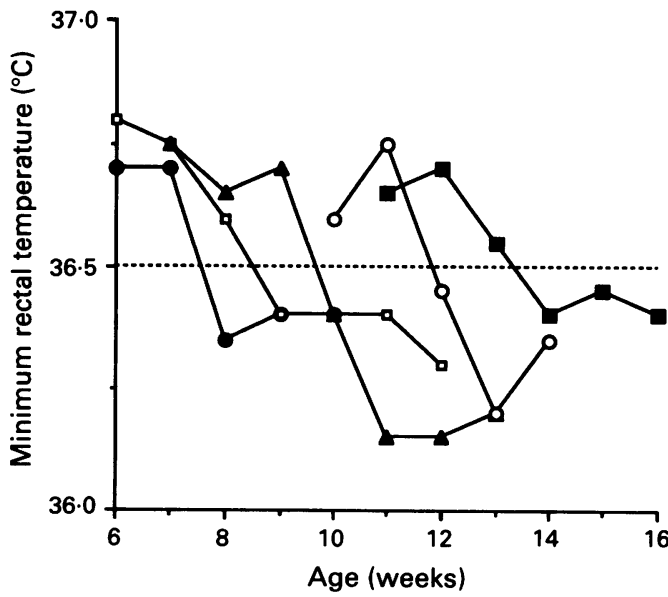

Figure 2 Minimum rectal temperature at night at various ages in five infants not including that illustrated in fig 1 . The largest and smallest decreases in minimum rectal temperature between two weeks are illustrated.

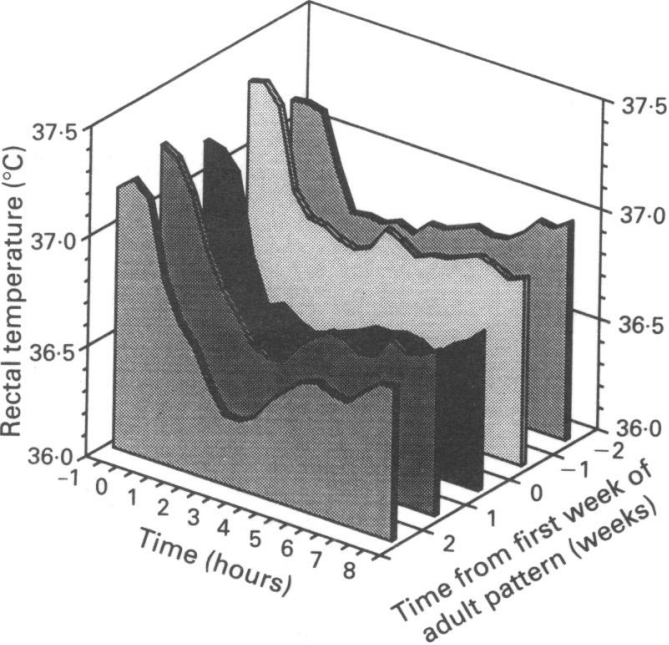

Figure 3 Averaged data from all 26 infants normalised to the first week in which minimum rectal temperature decreased to less than $36.5^{\circ} \mathrm{C}$. Standard errors (range $0.03-0.08^{\circ} \mathrm{C}$ ) are not shown for reasons of clarity.

There was a highly significant change in rectal temperature from the week before to the week in which temperature first decreased $(p<0.01$, Student's $t$ test), but no significant differences between the weeks after or before the change. Excluding periods of known illness only one of the 26 infants had a minimum rectal temperature greater than $36.5^{\circ} \mathrm{C}$ again after the week in which it first decreased to less than $36 \cdot 6^{\circ} \mathrm{C}$. On a few occasions, however, infants showed higher minimum temperatures the night before an illness such as upper respiratory tract infection.

We therefore chose the age at which the minimum rectal temperature first decreased to less than $36 \cdot 5^{\circ} \mathrm{C}$ as the age of 'maturation' of the night time temperature rhythm. The mean (SD) age was $11 \cdot 1(2 \cdot 5)$ weeks. Twelve of the infants matured between 7 and 11 weeks old (inclusive) and 14 between 12 and 16 weeks.

FACTORS AFFECTING THE AGE OF 'MATURATION' The infants who developed their mature temperature rhythms before 12 weeks old were significantly more likely to have been breast fed at birth $\left(\chi^{2}=5.26, p<0.025\right)$ and for at least six weeks $\left(\chi^{2}=5.56, p<0.025\right)$. The average chronological age of development of the rhythm for infants who were never breast fed was $12 \cdot 38$ $(0.52)$ weeks, whereas those breast fed for at least six weeks developed at 10.07 (0.74) weeks, a significant difference $(\mathrm{p}<0.01$, Student's $t$ test). Girls developed their rhythms significantly earlier than boys (average age for girls 10.23 (0.59) weeks; for boys (11.92 (0.44) weeks; $\mathrm{p}<0.05$, Student's $t$ test). There was a clear relation between the weekly rate of weight gain and the age of 'maturation' of the rhythm. Infants gaining weight most rapidly developed later (product moment correlation between average weekly weight gain and age of development $(r=0.513, \mathrm{p}<0.01)$

First children matured significantly earlier than second or subsequent children (average age for first children $10 \cdot 11(0 \cdot 74)$ weeks; for second and subsequent children $12.58(0.42)$ weeks; 
$\mathrm{p}<0.05$, Student's $t$ test). There was, however, no significant association between breast feeding and birth order. Infants sleeping prone were significantly more likely to develop their rhythms after 11 weeks old $\left(\chi^{2}=5 \cdot 51 ; p<0.025\right)$ though they were also significantly more likely to be second or subsequent children $\left(\chi^{2}=4 \cdot 34\right.$; $\mathrm{p}<0.05)$. The mothers of babies who matured before 12 weeks old were significantly older than those of babies who matured later $(p<0.05$, Student's $t$ test) and there was a strong trend, close to significance, for infants from families in the non-manual occupational groups to mature earlier than those from families in manual groups. There was no significant difference in room temperature or thermal insulation of clothing and wrapping between infants who developed before and after 11 weeks old. Infants who developed after 11 weeks old had not had more episodes of minor illness (such as upper respiratory tract infection) in the first weeks of life.

We have also analysed our data correcting for the small differences in gestational age between infants. All effects which were statistically significant with chronological ages remained significant when ages were corrected to a standard 40 week gestation.

\section{Discussion}

We have confirmed earlier observations that the minimum rectal temperature during sleep at night decreases abruptly around 11 weeks old. ${ }^{2}$ The transition from a minimum sleeping rectal temperature around $36.8^{\circ} \mathrm{C}$ to a minimum around $36.4^{\circ} \mathrm{C}$ was rapid. It was always complete between two weeks' recordings, and the few more frequent recordings we have suggested a time course of two or three days. Once the change occurred it did not revert unless the infant was ill. We saw only one occasion where the minimum sleeping rectal temperature of a healthy infant returned above $36.5^{\circ} \mathrm{C}$ after the change.

As we have reported previously the change in sleeping temperature usually coincided with an abrupt lengthening of the time infants spent in their cots before disturbing their parents. ${ }^{2}$ Not all infants, however, had settled into a regular sleep pattern at the time of 'maturation'. In particular, many breast fed infants were still feeding at night after the minimum rectal temperature decreased to less than $36.5^{\circ} \mathrm{C}$.

The abruptness and permanence of the change suggests that it is a physiological alteration on the endogenous mechanisms of temperature control. Its timing seems to result from an interaction between features of the infant and the way in which it is cared for. This leads to large individual differenes in timing with some infants 'maturing' at twice the age of others. We have identified a series of factors associated with early and late development. They mostly relate to one another and it is impossible at this stage to say which are primary and which secondary associations.

Breast fed babies 'matured' significantly earlier than bottle fed babies. This occurred even if babies were breast fed for just one or two weeks, suggesting that it was due to having received breast milk, rather than the process of breast feeding, which may have stopped by the time maturation occurred. Breast milk contains a variety of substances which are absent from formula feeds and which might interact with central mechanisms of development. Melatonin is a possibility. Girls matured significantly earlier than boys, though they were more likely to be breast fed. In the first weeks of life boys gained weight faster than girls, especially if bottle fed. Late maturation of temperature rhythms was associated with high rates of weight gain. We do not know whether the boys gained weight more quickly and matured temperature rhythms later because of some physiological consequence of masculinity, or because they were treated differently by parents, being more likely to be changed to bottle feeding. The relation between sleeping position and the timing of development is fascinating but inexplicable.

The remaining factors which were associated with early or late maturation were all features of the infant's family environment. Second babies developed later. Babies with older mothers developed earlier. Perhaps some feature of the general household environment such as the amount of parental contact or sensory stimulation affects the processes of development.

It is clear, however, that the development of something as simple as the daily rhythm of body temperature is the result of a complex interaction between intrinsic and extrinsic factors which could in principle be changed by intervention should particular patterns of development be shown to affect morbidity or mortality.

The work was supported by the Wellcome Trust and the Foundation for the Study of Infant Deaths.

1 Wailoo MP, Petersen SA, Whittaker H, Goodenough P. Sleeping body temperature in 3-4 month old infants. Arch Dis Child 1989;64:596-9.

2 Lodemore MR, Petersen SA, Wailoo MP. The development of night time temperature rhythms over the six months of life. Arch Dis Child 1991;66:521-4. 